\title{
X-RAY STANDING WAVE STUDIES OF Si (111) CRYSTAL IMPLANTED BY Fe AND Ni IONS
}

\author{
I.A. Vartanjantz, M.V. Kovalchuk, A.N. Sosphenov, \\ Institute of Crystallography, Academy of Sciences of USSR \\ 117333, Moscow, Leninsky Prospect 59, USSR \\ J. Auleytner and J. Majewski \\ Institute of Physics, Polish Academy of Sciences \\ Al. Lotników 32/46, 02-668 Warszawa, Poland \\ (Received August 8, 1991; in final form November 8, 1991)
}

\begin{abstract}
The X-Ray Standing Wave Method (XRSW) was applied for the investigation of the silicon samples implanted with $80 \mathrm{keV} \mathrm{Fe}$ and $\mathrm{Ni}$ ions. The samples were measured by the XRSW method before and after annealing process. For theoretical calculations the two layer model was used. The analysis revealed that after annealing only a slight amount $(\sim 20 \div 30 \%)$ of the implanted atoms occupy the position of the Si crystal planes. The Rutherford backscattering (RBS) experiment that confirms the results obtained by the XRSW method was performed.
\end{abstract}

PACS numbers: 61.10.-i, 61.70.Sk

\section{Introduction}

One of the powerful experimental methods for obtaining structural information about impurities in the perfect crystals is the X-Ray Standing Wave Method (XRSW). Measuring the fluorescence yield from the foreign atoms while scanning in angle through a strong Bragg diffraction condition it is possible to localize, with high accuracy, the atom positions inside a crystal lattice. For the structural investigation of the various impurity atoms ( $\mathrm{As}, \mathrm{Bi}$ ) after implantation this method was used by a number of authors [1,2]. In the present paper XRSW method was applied for the analysis of the structure of thin subsurface layers of $\mathrm{Si}$ obtained by $\mathrm{Fe}$ and $\mathrm{Ni}$ ions implantation. Investigation of the interaction of the transition metals with the Si bulk crystal is quite interesting from the point of view of the gettering phenomenon. 


\section{The fundamentals of the XRSW method}

Let us briefly remind the principles of the XRSW method (for details see e.g. [3-5]). For the dynamical case of the Bragg diffraction a standing wave which is generated by the interference of the incident $E_{0}(\theta)$ and reflected $E_{\mathrm{h}}(\theta)$ beams arises. Its period is equal to the period of the diffraction planes. A change of the $\mathrm{X}$-rays incidence angle $\theta$ within the region of the total reflection leads to the shift of the standing wave by one half of its period, which in its turn leads to a change of the fluorescence yield. This yield is determined by the position of atoms in the unit cell relative to nodes and antinodes of XRSW.

The general theory of the angular dependence of the secondary radiation yield in a crystal with a disturbed surface layer was developed in [6]. It was shown that in the common case of the arbitrary profile of deformation the intensity of the secondary radiation yield is determined not only by the specific features of the process (fluorescence, photoemission or some other kind of secondary radiation) but also by the dependence of the amplitudes $E_{0}(\theta, z)$ and $E_{\mathrm{h}}(\theta, z)$ on the depth $z$. If the crystal lattice distortion is known, then these amplitudes can be obtained, for example, by the numerical solution of the Takagi-Taupin equation $[6,7]$.

Let us consider the simplest situation when the impurity atoms-do not disturb the host crystal. Then for the amplitudes of the wave fields in the volume their values in the ideal crystal may be taken. These amplitudes decline exponentially with the increasing depth $z$, with the rate of decline determined by the interference absorption coefficient $\mu(\theta)$. In the case of the symmetrical Bragg geometry of diffraction for $\sigma$-polarized beam the intensity of the fluorescence yield from the atoms of type $\nu$ is described by the formula [4-6]:

$$
\begin{aligned}
& I^{\nu}(\theta)=I_{0}^{\nu} \chi^{\nu}(\theta) \int_{0}^{\infty} \mathrm{d} z P^{\nu}(z) \exp [-\mu(\theta) z], \\
& \chi^{\nu}(\theta)=1+\frac{\left|E_{\mathrm{h}}(\theta)\right|^{2}}{\left|E_{0}(\theta)\right|^{2}}+2 \frac{\left|E_{\mathrm{h}}(\theta)\right|}{\left|E_{0}(\theta)\right|} f_{\mathrm{c}}^{\nu} \cos \left[\alpha(\theta)+\phi_{\mathrm{c}}^{\nu}\right], \\
& f_{\mathrm{c}}^{\nu}=\left|S_{\mathrm{h}}^{\nu}\right| \exp \left(-M_{\mathrm{h}}^{\nu}-W_{\mathrm{h}}^{\nu}\right), \quad \phi_{\mathrm{c}}^{\nu}=h u^{\nu}+\omega,
\end{aligned}
$$

where $\alpha(\theta)$ is the phase of the complex ratio $E_{\mathrm{h}}(\theta) / E_{0}(\theta),\left|S_{\mathrm{h}}^{\nu}\right|$ and $\omega$ are the amplitude and phase of the effective structure factor for the implanted atoms of the type $\nu, \exp \left(-M_{\mathrm{h}}^{\nu}\right)$ and $\exp \left(-W_{\mathrm{h}}^{\nu}\right)$ are the thermal and the static Debye-Waller factors, describing the random displacement of atoms from their equilibrium position in a perfect crystal, $h$ is the reciprocal-lattice vector, $u^{\nu}$ is the displacement of the implanted atoms from the substitutional site in a host crystal. The function $P^{\nu}(z)=\exp \left(-\mu_{\mathrm{yi}}^{\nu} z\right)$ describes the probability of the photon penetrating from the depth $z$ to the crystal surface, $\mu_{y i}^{\nu}=1 / L_{\mathrm{yi}}$ is proportional to the cross-section of the photoelectric absorption, $L_{y i}$ is the escape depth of the fluorescence radiation. Moreover, if the depth of the implanted layer $L$ satisfies the condition $L \ll L_{\mathrm{ex}}, L_{\mathrm{yi}}$, where $L_{\mathrm{ex}}$ is the extinction lenghth (in this case, obviously, the 
yield of the fluorescence radiation is determined by the depth $L$ ), the intensity of the fluorescence yield is equal to

$$
I^{\nu}(\theta)=I_{0}^{\nu} \chi^{\nu}(\theta) L\left\{1-0.5\left[\mu_{y i}^{\nu}+\mu(\theta)\right] L\right\} .
$$

According to Eqs. $(1,2)$ from the shape of the fluorescence angular dependence curve it is possible to determine two parameters characterizing different aspects of the impurity atom structure in the implanted subsurface layer with a thickness $L$. These are the so-called coherent position $\phi_{c}^{\nu}$, characterizing the position of the impurity atoms with respect to the diffraction planes of the crystal, and the coherent fraction $f_{c}^{\nu}$, which describes the displacement of the atoms from the mean position. If these parameters are known, then, according to Eq. (2), it is possible to determine the thickness of implanted layer $L$.

In this paper thin subsurface layers of $\mathrm{Si}$ after implantation and annealing are investigated. It is reasonable to suppose that just after implantation the implanted atoms occupy chaotic position in the Si lattice. So, in this case the coherent fraction $f_{\mathrm{c}}^{\nu}$ must be equal to zero and the shape of fluorescence curve $I^{\nu}(\theta)$ must be the same as the rocking curve $P_{\mathrm{r}}(\theta)=\left|E_{\mathrm{h}}(\theta)\right|^{2} /\left|E_{0}(\theta)\right|^{2}$ (so, according to Eq. (2), it would be possible to determine the depth $L$ ). After annealing a part of the implanted atoms would occupy the substitutional sites and this would change the shape of the fluorescence curve and, hence, the value of the coherent fraction $f_{\mathrm{c}}^{\nu}$.

The situation is quite different if the condition $L_{\mathrm{yi}} \gg L_{\mathrm{ex}}$, is fulfilled (in this case the fluorescence radiation is collected from the volume determined by $\left.L_{y i}\right)$. This situation is realized, for example, for the $\mathrm{Si} K_{\alpha}$ fluorescence yield of $\mathrm{Si}$ lattice. The intensity of the secondary radiation curve turns out to be proportional to $\left(1-P_{\mathrm{r}}(\theta)\right)$ and the standing wave structure is hardly evident on the borders of the total reflection region (the so-called extinction effect [3-6]). In this case, all radiation absorbed in the crystal, the amount of which, following the law of conservation of energy, is equal to $\left(1-P_{\mathbf{r}}(\theta)\right)$, gives rise to a fluorescence signal.

\section{The sample preparation and experiment}

The $25 \times 25 \mathrm{~mm}^{2}$ and $10 \mathrm{~mm}$ thick (111) oriented crystal samples were cut off from a bulk of a dislocation free silicon crystal. One of the surfaces of each sample was mechanically polished to obtain mirror plane and then the whole sample was etched in the etching solution. The crystal samples perfection was checked using plane wave Lang reflection topography. The samples of Si (111) crystals were then implanted by $80 \mathrm{keV} \mathrm{Fe}$ (sample No. 1) and $\mathrm{Ni}$ (sample No. 2) ions with the dose $5 \times 10^{15} / \mathrm{cm}^{2}$.

After implantation the samples were measured using XRSW method and then annealed. The implanted samples were annealed at $750^{\circ} \mathrm{C}$, in $\mathrm{N}_{2}$ atmosphere. The annealing process was performed as follows:

$30 \mathrm{~min}$ : from room temperature to $750^{\circ} \mathrm{C}$,

45 min: at $750^{\circ} \mathrm{C}$,

$60 \mathrm{~min}$ : from $750^{\circ} \mathrm{C}$ to $500^{\circ} \mathrm{C}$, 
5 hours: from $500^{\circ} \mathrm{C}$ to room temperature.

After this annealing both samples were measured by XRSW method once again. The sample implanted with $\mathrm{Ni}$ ions was additionally annealed at $900^{\circ} \mathrm{C}$ with the same annealing process.

The X-ray standing wave experiment was carried out using the double-crystal non-dispersive scheme of symmetrical diffraction on the base of multicrystal spectrometer, which was designed and built in the A.V. Shubnikov Institute of Crystallography, USSR Academy of Sciences (for details see for example [5]). For Si (111) crystal planes $\mathrm{Cu} K_{\alpha}$ radiation (for registration of $\mathrm{Fe} K_{\alpha}$ yield) and $\mathrm{Cu} K_{\beta}$ radiation (for Ni $K_{\alpha}$ yield) were used (the energy of $\mathrm{Cu} K_{\alpha}$ is not high enough for $\mathrm{Ni} K_{\alpha}$ line excitation). The fluorescence radiation was registered by a $\mathrm{Si}(\mathrm{Li})$ solid state detector with energy resolution $200 \mathrm{eV}$. The fluorescence yield from implanted atoms with the dose $5 \times 10^{15} / \mathrm{cm}^{2}$ is very small (less than one registered pulse per second), with the ordinary source for measuring such doses it is usually necessary to use a synchrotron radiation source). In order to obtain reliable results precise data collection was used. The data collection system consists of an accurate torsion goniometer moved by a piezoceramic and a feedback loop for the temperature change compensation. The rocking and fluorescence yield curves were measured using the computer controlled multichannel analyzer. This system gives the possibility to obtain the two-dimensional data block (angle-energy) for choosing the energy range and for the preliminary calculations.

\section{Results and discussions}

The preliminary amplitude fluorescence analysis of the investigated crystals has revealed the following: the sample No. 1 contained Fe atoms only, the sample No. 2 (nominally implanted by $\mathrm{Ni}$ ions) contained $\mathrm{Ni}$ atoms and a small amount ( $5 \%$ of $\mathrm{Ni}$ atoms) of $\mathrm{Fe}$ atoms. Due to a small amount of $\mathrm{Fe}$ atoms in the sample No. 2 the XRSW experiment was carried out only on Ni atoms.

In Fig. 1a, b the rocking and Si $K_{\alpha}$ fluorescence curves for investigated crystals before annealing are presented (the theoretical curves are also shown in Fig. 1). Here, for the theoretical calculations of the rocking and Si $K_{\alpha}$ fluorescence curves, a model of an ideal crystal was used. Quite satisfactory agreement between the theoretical and experimental curves indicates good structural quality of the crystal bulk even after implantation. Slight asymmetric behaviour of the curve Eq. (1b) is due to the influence of the extinction effect [3-5]. The shape of the rocking curve for both samples does not change after annealing which indicates the stable quality of the host crystal.

In Fig. 2 the results of the Fe $K_{\alpha}$ fluorescence yield from the crystal No. 1 before annealing are presented. The experimental curve is quite similar to the rocking curve. As it was discussed before this is due to the fact that the implanted atoms occupy random positions in the lattice $\left(f_{\mathrm{c}}^{\mathrm{Fe}}=0\right)$. This is just the situation described by Eqs. (1b, 2). Taking for computer simulations for $E_{0}(\theta), E_{\mathrm{h}}(\theta)$ and $\mu(\theta)$ their values in the ideal crystal and varying the thickness of the implanted layer according to Eq. (2) the best result was obtained for $L=2000 \pm 500 \AA$ 

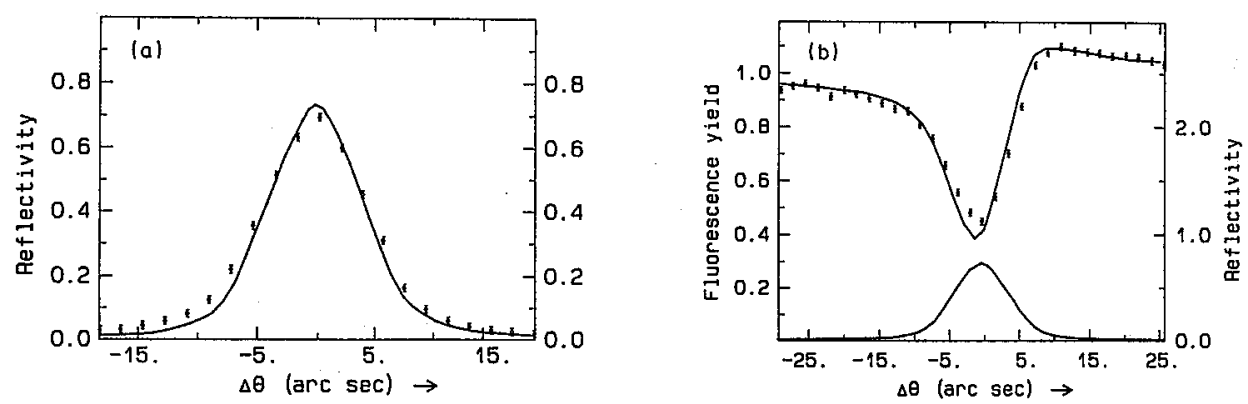

Fig. 1. Reflectivity (a) and Si $K_{\alpha}$ fluorescence yield (b) for $\operatorname{Si}(111)$ reflection $\mathrm{Cu} K_{\alpha}$ radiation (points - experiment, solid line - theoretical curve).

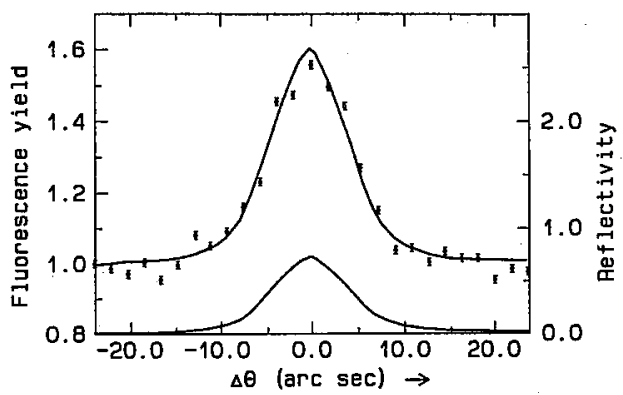

Fig. 2. Fluorescence $\mathrm{Fe} K_{\alpha}$ yield from the sample No. 1 before annealing. Solid line theoretical curve for the amorphous layer of Fe atoms (coherent fraction $f_{c}^{\mathrm{Fe}}=0$ ) with the thickness $L=2000 \AA$.

(a $\chi^{2}$-fit of the experimental data is also shown in Fig. 2). The same result was obtained for the Ni implanted sample No. 2.

Figure 3 shows $\mathrm{Fe} K_{\alpha}$ fluorescence yield for the sample No. 1 after annealing at $750^{\circ} \mathrm{C}$. In this case a part of the implanted atoms occupies the substitutional sites and the coherent fraction $f_{c}^{\nu}$ is not equal to zero anymore. Moreover, the implanted part of the crystal may have undergone some relaxation. So, more careful theoretical treatment is necessary in this case. For theoretical fitting a two layer model was used. The first disturbed layer containing implanted Fe atoms is the one that gives contribution to the secondary emission yield (for the profile of implanted atoms a uniform model was used). The second one is a thick ideal Si substrate. In the first layer besides the position of the $\mathrm{Fe}$ atoms relatively to $\mathrm{Si}$ atom planes the relaxation of this layer after annealing was taken into account. For the values of the amplitudes $E_{0}(\theta, z), E_{\mathrm{h}}(\theta, z)$ and for the intensity of the fluorescence yield an analytical solution obtained in [8] was used. Taking for computer simulations the thickness of the implanted layer $L=2000 \AA$ the best results for the coherent fraction $f_{\mathrm{c}}^{\mathrm{Fe}}=0.20 \pm 0.05$ and the lattice relaxation $\Delta d / d=(-3.8 \pm 0.6) \times 10^{-4}$ were obtained (for the results of the theoretical fitting see Fig. 3). The results of 

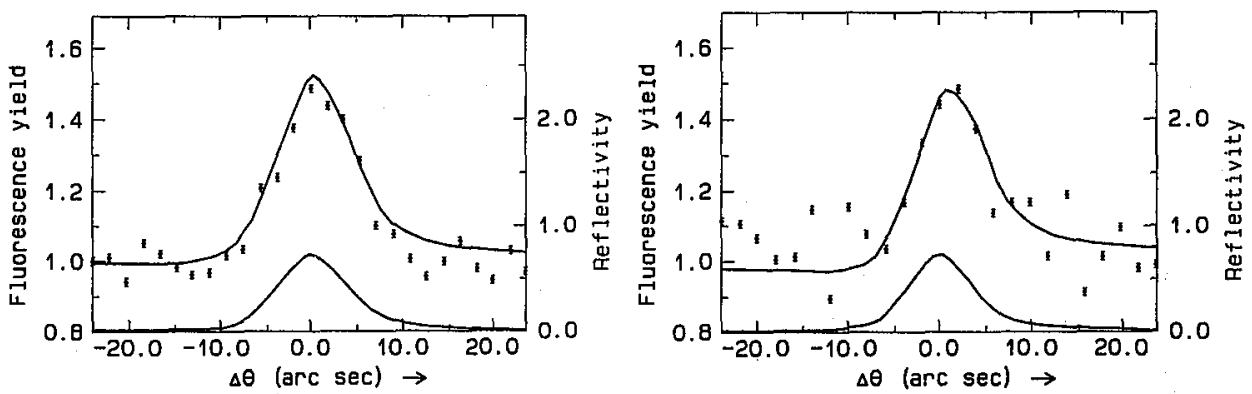

Fig. 3. Fluorescence Fe $K_{\alpha}$ yield from the sample No. 1 after $750^{\circ} \mathrm{C}$ annealing. Solid line - theoretical curve for the two layer model with the coherent fraction $f_{c}^{\mathrm{Fe}}=0.2$ and the surface relaxation $\Delta d / d=-3.8 \times 10^{-4}$.

Fig. 4. Fluorescence $\mathrm{Ni} K_{\alpha}$ yield from the sample No. 2 after the second $900^{\circ} \mathrm{C}$ annealing. Solid line - theoretical curve for the two layer model with the coherent fraction $f_{\mathrm{c}}^{\mathrm{Ni}}=0.3$ and the surface relaxation $\Delta d / d=-3.2 \times 10^{-4}$.

the XRSW experiments show that annealing conditions used have only slightly increased the amount of Fe atoms $(\sim 20 \%)$ that occupies the position of Si crystal planes.

In Fig. 4 the curve of $\mathrm{Ni} K_{\alpha}$ fluorescence yield obtained from the sample No. 2 after the second annealing at $900^{\circ} \mathrm{C}$ is shown (after the first annealing at $750^{\circ} \mathrm{C}$ the fluorescence curve from this sample was just the same as after implantation). The theoretical treatment with the same two layer model gives the following results for the coherent fraction: $f_{c}^{\mathrm{Ni}}=0.30 \pm 0.08$ and the lattice relaxation $\Delta d / d=$ $(-3.2 \pm 0.9) \times 10^{-4}$ (the same layer thickness $L=2000 \AA$ was used). So in the case of the sample No. 2 a little bit more amount of $\mathrm{Ni}$ atoms $(\sim 30 \%)$ occupies the lattice plane position. This may be the result of the more powerful annealing.

A few concluding remarks are necessary. In this paper the possibilities of the XRSW method for the investigation of the implanted layers of the crystals were demonstrated. It is interesting to compare these results with some other independent methods. Rutherford backscattering (RBS) experiments on the same crystals were performed. For the maximum depth of the impurity atoms $L_{\max } \approx$ $1700 \div 2200 \AA$ was obtained. For amorphization state RBS experiments give accordingly: $\sim 100 \%$ before annealing (all impurity atoms in chaotic positions) and $\sim 65 \%$ after annealing ( $35 \%$ of impurity atoms at substitutional sites). So, the results of the RBS experiments coincide to one order of magnitude with XRSW experiments.

These experimental (XRSW and RBS) values obtained on real crystals were also compared with the results of the theoretical calculations using PRAL computer program [9]. It gives for the projected range and longitudinal straggling of $\mathrm{Fe}$ and $\mathrm{Ni}$ ions of $80 \mathrm{keV}$ energy the following values: $668 \AA$ and $220 \AA$ (Fe), $628 \AA$ and $206 \AA(\mathrm{Ni})$. Results of these calculations give a value for the depth of the implanted ions more than two times lower. Unfortunately we cannot discuss this 
discrepancy between experimental and theoretical values because of the lack of concrete information about the detailes of PRAL calculation.

The simplest uniform model for the profile of impurity atoms and deformation was used in this paper. For investigation of the details of the profile (taking for example Gauss or some other model) it is necessary to perform XRSW experiments with higher statistics. For example, utilization of rotating anode or synchrotron radiation source would give better statistics for the same amount of impurity atoms.

\section{Acknowledgements}

The authors are grateful for support and helpful discussions on the problem to Dr. S.I. Zheludeva and to Dr. A.Yu. Kasimirov. The authors wish to thank Mr. J. Kaczanowski and Mr. S. Kwiatkowski from the Institute of Nuclear Problems, Świerk, Poland for RBS measurements which have been very helpful for testing the results obtained by XRSW.

\section{References}

[1] J.A. Golovchenko, B.W. Batterman, W.L. Brown, Phys. Rev. B 10, 4239 (1974).

[2] N. Hertel, G. Materlik, J. Zegenhagen, Z. Phys. B, Condens. Matter 58, 199 (1985).

[3] B.W. Batterman, Phys. Rev. A 133, 759 (1964).

[4] B.W. Batterman, H. Cole, Rev. Mod. Phys. 36, 681 (1964).

[5] M.V. Kovalchuk, V.G. Kohn, Usp. Fiz. Nauk 149, 69 (1986) (Sov. Phys.-Usp., 29, 426 (1986)).

[6] A.M. Afanasev, V.G. Kohn, Zh. Eksp. Teor. Fiz. 74, 300 (1978) (Sov. Phys.-JETP 47, 154 (1978)).

[7] V.G Kohn, M.V. Kovalchuk, Phys. Status Solidi A 64, 359 (1981).

[8] M.V. Kovalchuk, V.G. Kohn, E.F. Lobanovich, Fiz. Tverd. Tela, 27, 3379 (1985).

[9] J.F. Ziegler, J.P. Biersack, U. Littmark, The Stopping and Range of Ions in Matter, Pergamon Press, New York 1985. 